\title{
Multihop Free-Space Optical Communications Over Strong Turbulence Channels
}

\author{
Theodoros A. Tsiftsis*, Harilaos G. Sandalidis ${ }^{\dagger}$, George K. Karagiannidis ${ }^{\ddagger}$, and Nikos C. Sagias ${ }^{\S}$ \\ *Department of Electrical \& Computer Engineering, University of Patras, Rion, 26500 Patras, Greece, Email: tsiftsis@ee.upatras.gr \\ ${ }^{\dagger}$ Greek Ombudsman, 5 Hadjiyanni Mexi str., 11528 Athens, Greece, Email: sandalidis@ hotmail.com \\ ${ }^{\ddagger}$ Department of Electrical \& Computer Engineering, Aristotle University of Thessaloniki, 54124 Thessaloniki, Greece, Email: geokarag@auth.gr \\ §Institute of Informatics \& Telecommunications, NCSR “Demokritos", Agia Paraskevi, 15310 Athens, Greece, Email: nsagias@ieee.org
}

\begin{abstract}
In this paper, we study the performance of multihop free-space optical (FSO) wireless systems over turbulenceinduced fading channels. The analysis is carried out for systems employing amplify-and-forward (AF) or decode-and-forward (DF) relays and for turbulence channels which can be modeled by the Gamma-Gamma distribution. An exact analytical expression for the end-to-end outage probability of AF systems is obtained, while a closed-form expression of DF systems is derived. The average bit-error probability of a dual-hop FSO system employing a DF relay is studied as a special case. Numerical examples are also presented to illustrate the proposed analysis and to further investigate the effects of the turbulence severity on the multihop FSO systems' performance.
\end{abstract}

\section{INTRODUCTION}

Free-space optical (FSO) communications has gained significant research attention in recent years. Channels in FSO systems have wider bandwidth and therefore are able to support more users compared to radio frequency (RF) ones. However, the existence of atmospheric turbulence, which takes place because of the variations in the refractive index due to inhomogeneities in temperature and pressure fluctuations, can degrade the performance of the communication system mainly over distances of $1 \mathrm{~km}$ or longer [1]. The reliability of a FSO link can be determined if we use an effective probabilistic model for the turbulence. It has been observed that for weak fluctuations, the distribution of received intensities is close to a log-normal. For strong turbulence conditions Al-Habash et al. proposed the Gamma-Gamma distribution, which is a multiplicative random process and used to model both smallscale and large-scale fluctuations [2].

The performance analysis of point-to-point coded FSO links employing on-off keying (OOK) with intensity modulation/direct direction (IM/DD), over weak or strong turbulence conditions, has been an important field of research in recent years with the works of Zhu and Kahn in [3], [4] and Uysal et al. in [5], [6]. In order to increase the reliability of a FSO link, an important technique has been introduced in the literature, where the distance between the transmitter and the receiver is scaling down via multihop routing [7]. This technique is common in various wireless RF communication systems where relayed transmission is providing broader and more efficient coverage. Recently, a bit-error rate (BER) analysis for decode-and-forward (DF) and amplify-and-forward (AF)
FSO relaying systems has been presented in [8]. Specifically, the authors in [8] compared simulation results for multihop DF relaying systems to single-hop optical links. Furthermore, the AF relays use less complex circuitry because they just amplify and forward the incoming signal without performing any sort of decoding. On the other hand, the DF relays decode the signal and then transmit the detected version to the destination ones. The outage performance of multihop systems employing relays was extensively studied for the case of RF wireless communications [9]-[11]. However, to the best of the authors' knowledge, the outage performance of multihop FSO communication systems has never been addressed in the past.

In this paper, the outage probability of a multihop FSO communication system with $\mathrm{AF}$ or DF relays over strong turbulence fading channels, is studied. For the case of AF systems, we assume channel state information-based relays with knowledge of the channel in the preceding hop. Closedform expression for the moment generating function (MGF) of the inverse end-to-end signal-to-noise ratio (SNR) is derived for the case of AF relays. Based on this expression, the outage probability for independent and non-identical Gamma-Gamma distributed channels is evaluated numerically via inverse Laplace transform. For the case of DF relays, the end-to-end outage probability is a product of the cumulative distribution functions (CDFs) of the point-to-point optical links. Moreover, a dual-hop FSO system with DF relay is considered, and its average BER performance is investigated.

The remainder of this paper is organized as follows. Section II introduces the system and channel model assumptions. Section III is dedicated to the outage performance analysis. Next, the average error probability of a dual-hop FSO system is examined in Section IV. Finally some numerical examples and concluding remarks are provided in Sections V and VI, respectively.

\section{System And Channel Model}

\section{A. System Model}

A multihop FSO wireless communication system where IM/DD links-hops using OOK is considered (see Fig. 1). The modulated light source communicates with the destination terminal through $R_{i}, i=1,2, \ldots N-1$ optical transceivers, 
which act as relays-nodes all being in equidistance $L$. Therefore, there are $N$ point-to-point propagation links before the light arriving to its destination. The received signal at each intermediate relay and at the final destination, can be written as

$$
y_{N}=\prod_{i=1}^{N} g_{i-1} s_{i} x+\sum_{i=1}^{N} n_{i}\left(\prod_{t=i+1}^{N} g_{t-1} s_{t}\right), g_{0}=1
$$

where $s_{i}=\eta I_{i}$ denotes the instantaneous intensity gain of the $i$ th hop, $x \in\{0,1\}$ the OOK modulated signal, $g_{i}$ is the gain of the $i$ th relay, $n_{i}$ is the additive white Gaussian noise (AWGN) signal at the input of the $i$ th relay with power spectral density $N_{0}, \eta$ the effective photo-current conversion ratio of the receiver and $I_{i}$ the turbulence-induced light intensity at the $i$ th hop. Then, the equivalent end-to-end SNR, i.e., the SNR at the receiver, can be written as [9]

$$
\mu=\frac{\prod_{i=1}^{N} s_{i}^{2} g_{i-1}^{2}}{\sum_{i=1}^{N} n_{i}\left(\prod_{j=i+1}^{N} g_{j-1}^{2} s_{j}^{2}\right)}
$$

One choice for the gain was proposed in [12] for RF links as $g_{i}^{2}=1 /\left(s_{i}^{2}+N_{0}\right)$. This gain aims to limit the instantaneous output power of the relay if the channel gain of the preceding hop, $s_{i}$, is low. By substituting the above gain in (2), the derived equivalent SNR at the receiver can be expressed by [9, eq. (2)]. From (2), the derivation of the distribution of $\mu$ is very difficult, if not possible, to be extracted. However, by setting the gain as the inverse of the fading state $g_{i}^{2}=1 / s_{i}^{2}$, the relay just amplifies the incoming signal with the inverse of the channel of the previous hop, regardless of the noise of that hop. As mentioned in [9], such a kind of relay serves as benchmark for all practical multihop RF systems employing AF relays. Equivalently, the end-to-end SNR of the considered multihop FSO system can be extracted as

$$
\mu=\left(\sum_{i=1}^{N} \frac{1}{\mu_{i}}\right)^{-1}
$$

where $\mu_{i}=\eta^{2} I_{i}^{2} / N_{0}$ is the instantaneous electrical SNR of the $i$ th hop.

\section{B. Channel Model}

To characterize the FSO channel from a communication theory point-of -view, a statistical representation of turbulenceinduced fading has to be given. Several probability density functions (PDFs) to describe strong turbulence effects have been proposed included the $K$ and $I-K$ distributions, however an effective statistical model has been proposed by [1], [2] to describe both small-scale and large-scale atmospheric fluctuations, the Gamma-Gamma model. This model factorizes the irradiance as the product of two independent random processes each one having a Gamma PDF. The derivation of

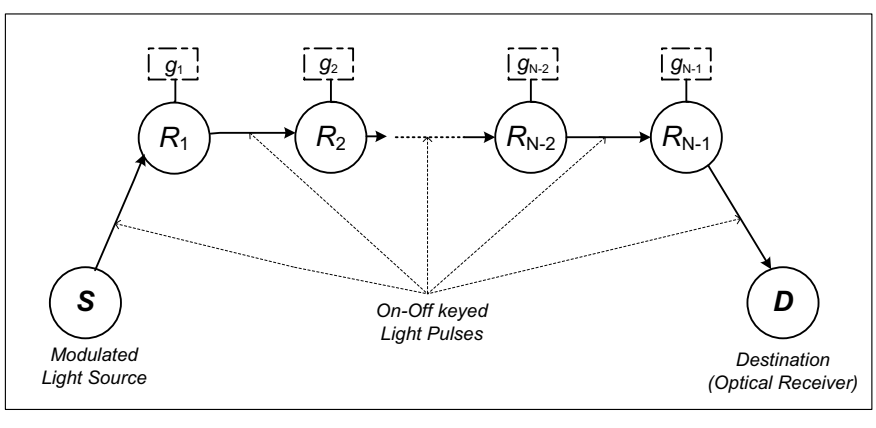

Fig. 1. Multihop FSO communication system.

the Gamma-Gamma distribution is given in [2] resulting in

$$
\begin{aligned}
f_{I_{i}}(I)=\frac{2(\alpha \beta)^{(\alpha+\beta) / 2}}{\Gamma(\alpha) \Gamma(\beta) \bar{I}_{i}} & \left(\frac{I}{\bar{I}_{i}}\right)^{\frac{\alpha+\beta}{2}-1} \\
& \times K_{\alpha-\beta}\left[2 \sqrt{\alpha \beta\left(\frac{I}{\bar{I}_{i}}\right)}\right], I \geq 0
\end{aligned}
$$

where $\bar{I}_{i}$ denotes the average irradiance of the $i$ th channel, $K_{\nu}[\cdot]$ is the $\nu$ th-order modified Bessel function of the second kind, defined in [13, eq. (8.432)], and $\alpha, \beta$ are the effective number of small-scale and large-scale eddies of scattering environment, where the two scales are implemented mathematically by inserting heuristic spatial-frequency filters into weak-turbulence integrals for scintillation index. According to [2], [14]

$$
\begin{aligned}
& \alpha=\left[\exp \left(\frac{0.49 \beta_{0}^{2}}{\left(1+0.18 d^{2}+0.56 \beta_{0}^{12 / 5}\right)^{7 / 6}}\right)-1\right]^{-1} \\
& \beta=\left[\exp \left(\frac{0.51 \beta_{0}^{2}}{\left(1+0.9 d^{2}+0.62 d^{2} \beta_{0}^{12 / 5}\right)^{5 / 6}}\right)-1\right]^{-1}
\end{aligned}
$$

where $\beta_{0}^{2}=0.5 C_{n}^{2} k^{7 / 6} L^{11 / 6}$ is the Rytov variance for a plane wave in weak scintillation theory and $d=\left(k D^{2} / 4 L\right)^{1 / 2}$ the parameter characterizing the receiver aperture diameter and hence aperture averaging effects. Here, $k=2 \pi / \lambda$ is the optical wave number, $\lambda$ is the wavelength, $C_{n}^{2}$ denotes the index of refraction structure parameter and $D$ is the diameter of the receiver collecting lens aperture.

Hence, after a simple power transformation of the random variable (RV) $I_{i}$, the PDF of the electrical SNR of each hop, $\mu_{i}$, can be derived as

$$
\begin{aligned}
f_{\mu_{i}}(\mu)=\frac{(\alpha \beta)^{(\alpha+\beta) / 2}}{\Gamma(\alpha) \Gamma(\beta) \sqrt{\bar{\mu}_{i} \mu}} & \left(\sqrt{\frac{\mu}{\bar{\mu}_{i}}}\right)^{(\alpha+\beta) / 2-1} \\
& \times K_{\alpha-\beta}\left[2 \sqrt{\alpha \beta \sqrt{\frac{\mu}{\bar{\mu}_{i}}}}\right]
\end{aligned}
$$


where $\bar{\mu}_{i}=(\eta E[I])^{2} / N_{0}$ is the electrical average SNR, defined in [3].

By following the definition, $F_{\mu_{i}}(\mu)=\int_{0}^{\mu} f_{\mu_{i}}(z) d z$ and using (5), the derived integral for the CDF of the GammaGamma channel is not a tabulated one. In order to evaluate it, the CDF can be written as

$$
\begin{aligned}
& F_{\mu_{i}}(\mu)=\frac{(\alpha \beta)^{(\alpha+\beta) / 2}}{2 \Gamma(\alpha) \Gamma(\beta) \sqrt[4]{\bar{\mu}_{i}^{\alpha+\beta}}} \\
& \times \int_{0}^{\mu} z^{(\alpha+\beta) / 4-1} G_{0,2}^{2,0}\left[\frac{\alpha \beta}{\sqrt{\bar{\mu}_{i}}} z^{1 / 2} \mid \frac{\alpha-\beta}{2}, \frac{\beta-\alpha}{2}\right] d z
\end{aligned}
$$

where $K_{\nu}[\cdot]$ using [15, eq. (14)] is written in terms of the Meijer's G-function $G_{p, q}^{m, n}[\cdot]$ [13, eq. (9.301)]. Therefore, using [15, eq. (26)], a closed-form ${ }^{1}$ solution for (6) leads to

$$
\begin{aligned}
F_{\mu_{i}}(\mu) & =\frac{(\alpha \beta)^{(\alpha+\beta) / 2}}{\Gamma(\alpha) \Gamma(\beta)} \sqrt[4]{\left(\frac{\mu}{\bar{\mu}_{i}}\right) \alpha+\beta} \\
& \times G_{1,3}^{2,1}\left[\alpha \beta \sqrt{\frac{\mu}{\bar{\mu}_{i}}} \mid \begin{array}{c}
\frac{\alpha-\beta}{2}, \frac{\beta-\alpha}{2},-\frac{\alpha+\beta}{2}
\end{array}\right] .
\end{aligned}
$$

\section{Outage Probability}

\section{A. AF Relays}

The outage probability is defined as the probability that the instantaneous SNR falls below a specified threshold $\mu_{t h}$, which represents a protection value of the SNR above which the quality of the channel is satisfactory. In case of the system under consideration, the equivalent end-to-end outage probability can be expressed by

$$
\begin{aligned}
P_{\text {out }}\left(\mu_{t h}\right) & =\operatorname{Pr}\left(\mu \leq \mu_{t h}\right)=\operatorname{Pr}\left(\frac{1}{\mu} \geq \frac{1}{\mu_{t h}}\right) \\
& =1-\left.\mathcal{L}^{-1}\left(\frac{\mathcal{M}_{1 / \mu}(s)}{s}\right)\right|_{s=1 / \mu_{t h}}
\end{aligned}
$$

where $\mathcal{L}^{-1}(\cdot)$ is the inverse Laplace transform and $\mathcal{M}_{1 / \mu}(\cdot)$ is the MGF of $1 / \mu$. Using (3) and due to the independency of the turbulence-induced fading channels, $\mathcal{M}_{1 / \mu}(s)$ can be written as

$$
\mathcal{M}_{1 / \mu}(s)=\prod_{i=1}^{N} \mathcal{M}_{1 / \mu_{i}}(s) .
$$

Using (5) and the definition of the MGF of $1 / \mu_{i}$, i.e., $\mathcal{M}_{1 / \mu_{i}}(s)=\int_{0}^{\infty} e^{-s / x} f_{\mu_{i}}(x) d x$, yields

$$
\begin{aligned}
& \mathcal{M}_{1 / \mu_{i}}(s)=\frac{(\alpha \beta)^{(\alpha+\beta) / 2}}{2 \Gamma(\alpha) \Gamma(\beta) \sqrt[4]{\bar{\mu}_{i}^{\alpha+\beta}}} \int_{0}^{\infty} x^{(\alpha+\beta) / 4-1} \\
& \times G_{0,1}^{1,0}\left[\begin{array}{c|c}
\frac{s}{x} & -
\end{array}\right] G_{0,2}^{2,0}\left[\frac{\alpha \beta}{\sqrt{\bar{\mu}_{i}}} x^{1 / 2} \mid \frac{\alpha-\beta}{2}, \frac{\beta-\alpha}{2}\right] d x
\end{aligned}
$$

\footnotetext{
${ }^{1}$ Note, that the Meijer's G-function is a standard built-in function in most of the well-known mathematical software packages such as MAPLE, MATHEMATICA, and MATLAB. In addition, using [15, eq. (18)], the Meijer's G-function can be written in terms of the more familiar generalized hypergeometric functions [13, eq. (9.14.1)].
}

where the exponential function is written in terms of the Meijer's G-function [15, eq. (11)]. Hence, by applying [13, eq. (9.31.2)], (10) can be rewritten as

$$
\begin{aligned}
& \mathcal{M}_{1 / \mu_{i}}(s)=\frac{(\alpha \beta)^{(\alpha+\beta) / 2}}{2 \Gamma(\alpha) \Gamma(\beta) \sqrt[4]{\bar{\mu}_{i}^{\alpha+\beta}}} \int_{0}^{\infty} x^{(\alpha+\beta) / 4-1} \\
& \times G_{1,0}^{0,1}\left[\begin{array}{c|c}
\frac{x}{s} & -
\end{array}\right] G_{0,2}^{2,0}\left[\frac{\alpha \beta}{\sqrt{\bar{\mu}_{i}}} x^{1 / 2} \mid \frac{\alpha-\beta}{2}, \frac{\beta-\alpha}{2}\right] d x .
\end{aligned}
$$

The integral in (11) can be evaluated using [15, eq. (21)], resulting in

$$
\begin{aligned}
& \mathcal{M}_{1 / \mu_{i}}(s)=\frac{(\alpha \beta)^{(\alpha+\beta) / 2} s^{(\alpha+\beta) / 4}}{4 \pi \Gamma(\alpha) \Gamma(\beta) \sqrt[4]{\bar{\mu}_{i}^{\alpha+\beta}}} \\
& \times G_{0,5}^{5,0}\left[\frac{(\alpha \beta)^{2} s}{16 \bar{\mu}_{i}} \mid \frac{\alpha-\beta}{4}, \frac{\alpha-\beta+2}{4}, \frac{\beta-\alpha}{4}, \frac{\beta-\alpha+2}{4},-\frac{\alpha+\beta}{4}\right] .
\end{aligned}
$$

By substituting (12) in (9), $\mathcal{M}_{1 / \mu}(s)$ is derived in closed form. Consequently, the outage probability given in (8), can be evaluated using any numerical method for the inverse Laplace transform (e.g., as the one used in [9]).

\section{B. DF Relays}

When a multihop FSO communication system is employing DF relays, then the outage performance of the system depends on the outage probability of each hop. Hence, the outage probability is given by

$$
\begin{aligned}
P_{\text {out }}\left(\mu_{t h}\right) & =\operatorname{Pr}\left[\min \left(\mu_{i}\right) \leq \mu_{t h}\right]=1-\prod_{i=1}^{N} \operatorname{Pr}\left[\mu_{i} \geq \mu_{t h}\right] \\
& =1-\prod_{i=1}^{N}\left[1-F_{\mu_{i}}\left(\mu_{t h}\right)\right] .
\end{aligned}
$$

By substituting (7) in (13), $P_{\text {out }}\left(\mu_{t h}\right)$ can be derived in closed form.

\section{A Special Case: Average BER for Dual-Hop FSO SYSTEMS WITH DF RELAYS}

In this section, the average BER for a dual-hop FSO system with DF relay over independent and identically distributed (i.i.d.) Gamma-Gamma turbulence-induced fading channels, is presented. Equivalently to RF case, the overall conditional probability of error is given by [16, eq. (11.4.12)]

$$
\begin{aligned}
P_{b}^{(\text {total })}\left(E \mid \mu_{1}, \mu_{2}\right)= & P_{b}\left(E \mid \mu_{1}\right)+P_{b}\left(E \mid \mu_{2}\right) \\
& -2 P_{b}\left(E \mid \mu_{1}\right) P_{b}\left(E \mid \mu_{2}\right)
\end{aligned}
$$

which, when averaged over i.i.d. Gamma-Gamma RVs $\mu_{1}$ and $\mu_{2}$, reduces to

$$
P_{b}^{(\text {total })}(E)=2 P_{b}(E)-2 P_{b}^{2}(E)
$$

where $P_{b}(E)$ is the average BER per hop. 
In general, a union upper bound on the average BER is given in [17] as

$$
P_{b}(E) \leq \frac{1}{\pi} \int_{0}^{\pi / 2}\left[\left.\frac{1}{n} \frac{\partial}{\partial \mathrm{N}} T(D(\theta), \mathrm{N})\right|_{\mathrm{N}=1}\right] d \theta
$$

where $n_{c}$ is the number of information bits per transmission, $\mathrm{N}$ is an indicator depends on the number of bits in error, $T(D(\theta), \mathrm{N})$ is the transfer function associated with the state diagram of a particular trellis-coded modulation scheme and $D(\theta)$ is based on the derived pairwise error probability.

Using a convolutional code with rate $1 / 3$ and constraint length of 3 , the transfer function of this code is [18, eq. (8.2.6)]

$$
T(D(\theta), \mathrm{N})=\frac{D^{6}(\theta) \mathrm{N}}{\left[1-2 \mathrm{~N} D^{2}(\theta)\right]^{2}} .
$$

The exact $D(\theta)$ is given in analytical form by [6, eq. (24) $]^{2}$

$$
\begin{aligned}
D(\theta)=\frac{2(\alpha \beta)^{\frac{\alpha+\beta}{2}}}{\Gamma(\alpha) \Gamma(\beta)} & \int_{0}^{\infty} I^{\frac{\alpha+\beta}{2}-1} \exp \left(-\frac{\tau}{4} \frac{I^{2}}{\sin ^{2} \theta}\right) \\
& \times K_{\alpha-\beta}[2 \sqrt{\alpha \beta I}] d I
\end{aligned}
$$

where $\tau=E_{s} / N_{0}$ is defined as SNR per optical link with $E_{s}$ being the total transmitted energy from the source or the DF relay. Also, the noise can be modeled as AWGN with zero mean and variance $N_{0} / 2$, independent of the on/off state of the received bit. By applying [15, eq. (21)] in (18), a closed-form solution can be extracted as

$$
\begin{aligned}
& D(\theta)=\frac{2^{\alpha+\beta-1}}{2 \pi \Gamma(\alpha) \Gamma(\beta)} \\
& \times G_{4,1}^{1,4}\left[\frac{4 \tau}{(\alpha \beta)^{2} \sin ^{2} \theta} \mid \begin{array}{c}
\frac{1-\alpha}{2}, \frac{2-\alpha}{2}, \frac{1-\beta}{2}, \frac{2-\beta}{2} \\
0
\end{array}\right] .
\end{aligned}
$$

By substituting (19) in (16) and using (14), an upper bound for the average BER of the dual-hop FSO system can be easily evaluated.

\section{Numerical Results}

In Fig. 2, the end-to-end outage probability is plotted as a function of the normalized to outage threshold average SNR per hop, $\bar{\mu} / \mu_{t h}$, assuming i.i.d. Gamma-Gamma channels (i.e., $\left.\bar{\mu}_{i}=\bar{\mu}\right)$ for several values of $N$. The obtained results indicate that the outage performance of the system degrades with an increase of the number of hops and shows similar behavior with the RF multihop systems. Furthermore, Fig. 3 compares the outage probability of a triple-hop FSO system with AF relays to a triple-hop one with DF relays versus the number of hops. It is obvious that DF systems slightly outperformed compared to AF ones and the performance gap is increasing with an increase of $N$. This result is similar as in RF multihop communications. Finally, in Fig. 4, the average BER for a dualhop FSO using a DF relay, is plotted. Also, in order to compare its error performance with point-to-point links, we use the

\footnotetext{
${ }^{2}$ Note that in [6, eq. (24)], the power term $(\alpha+\beta) / 2$ is missing from the product $(\alpha \beta)$.
}

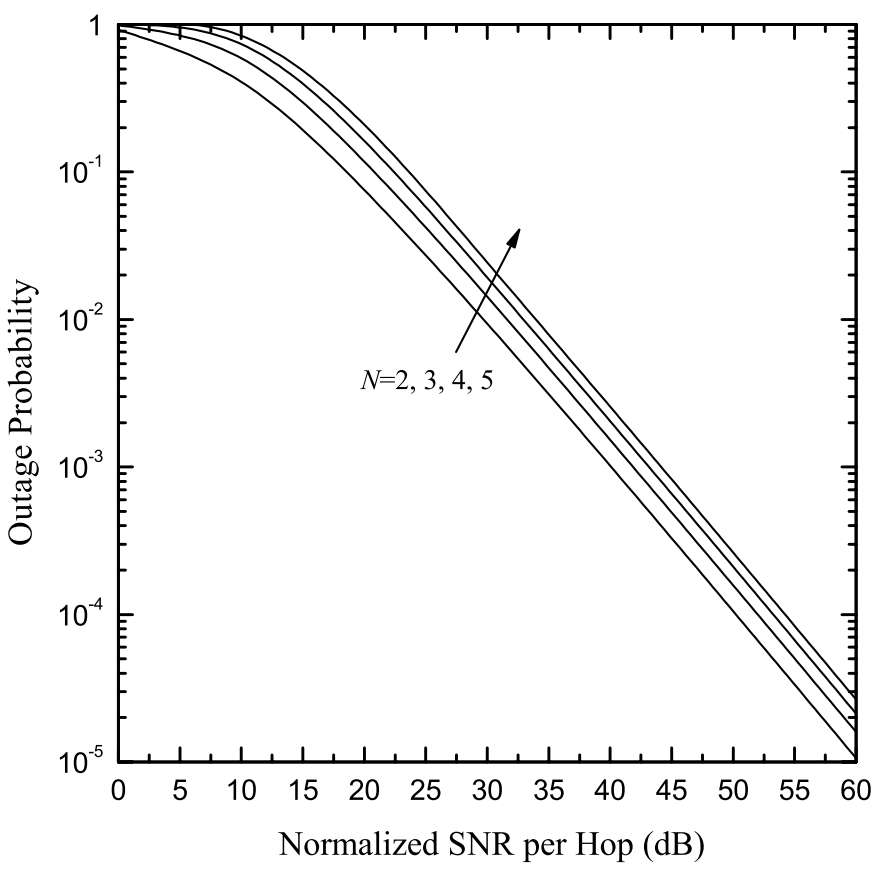

Fig. 2. End-to-end outage probability of an $N$-hop FSO system over i.i.d. Gamma-Gamma channels versus $\bar{\mu} / \mu_{t h}$ for several number of hops ( $\alpha=4, \beta=2$ for all hops).

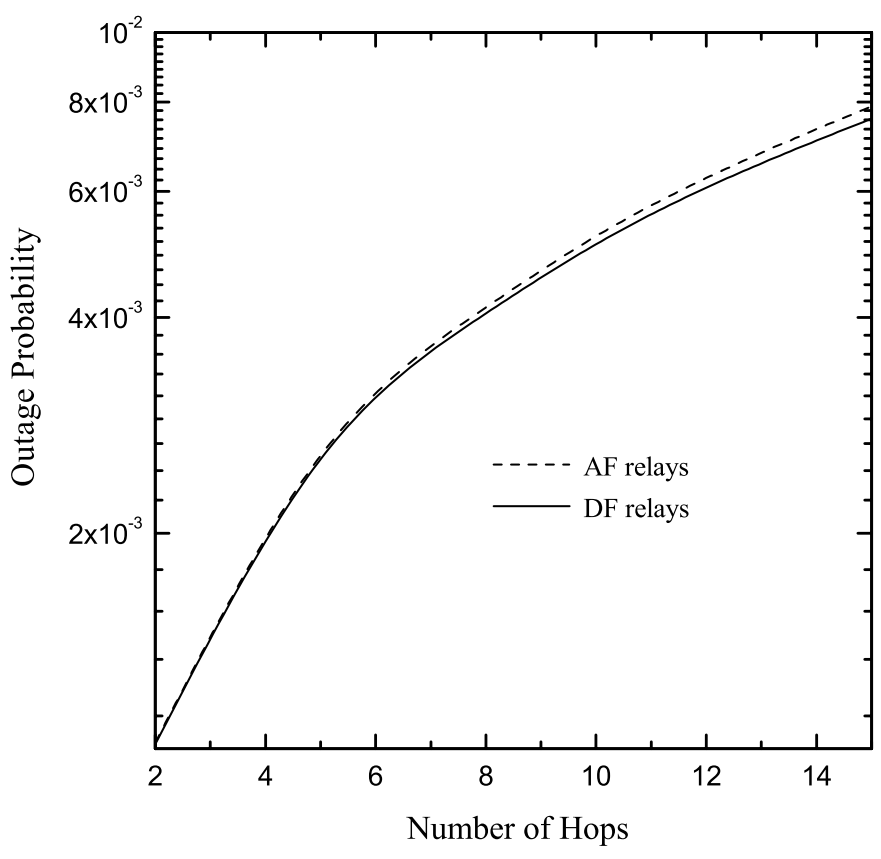

Fig. 3. Effect of increasing the number of hops on the end-to-end outage performance of a triple-hop FSO system using AF or DF relays over i.i.d. Gamma-Gamma channels $\left(\alpha=4, \beta=2\right.$ and $\bar{\mu} / \mu_{t h}=40 \mathrm{~dB}$ for all hops).

same values for the parameters that affect the system as the one used in [6]. Therefore, we assume wavelength $\lambda=1550 \mathrm{~nm}$ and the refraction index $C_{n}^{2}=1.7 \times 10^{-4}$. Also it is assumed $D \ll L$ leading to $d=0$ and hence no aperture averaging is possible. The distances between hops are equal and selected as $L=3000 \mathrm{~m}$ and $L=3400 \mathrm{~m}$ resulting in channel parameters 


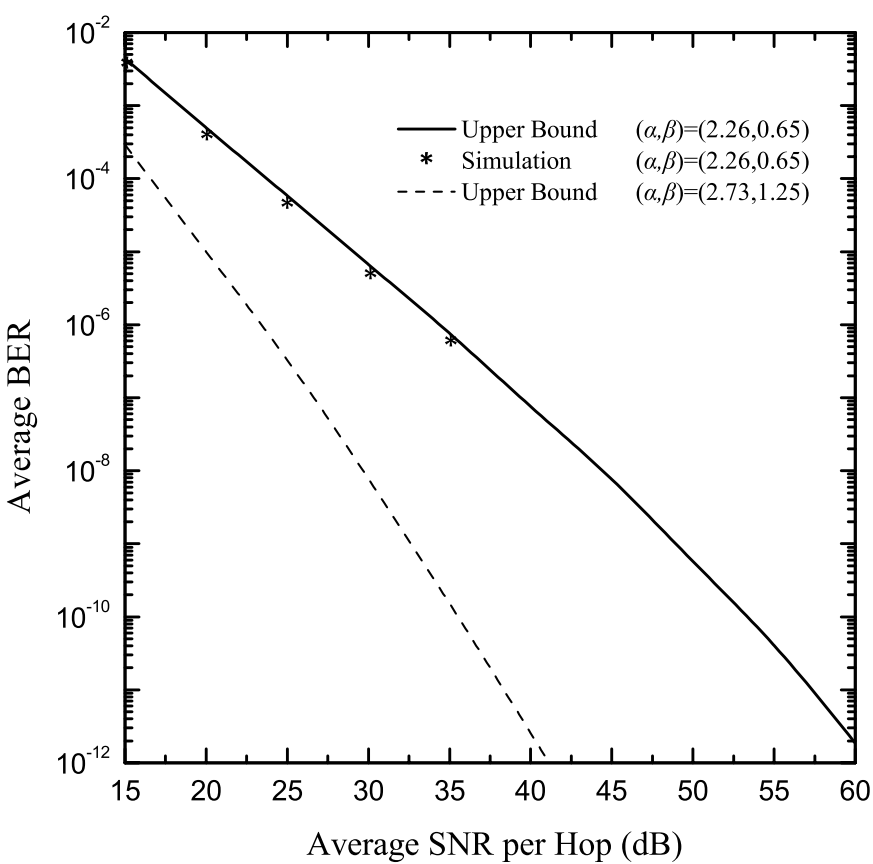

Fig. 4. Upper bounds on the average BER for a dual-hop FSO system with a DF relay over i.i.d. Gamma-Gamma channels versus average SNR per hop.

$(\alpha, \beta)=(2.73,1.25)$ and $(\alpha, \beta)=(2.26,0.65)$, respectively. It is observed that the dual-hop FSO system is outperformed compared to the single-hop optical links investigated in [6]. This result was also extracted in [8] via simulations. The simulation results depicted only for $\mathrm{BER} \approx 10^{-6}$ and for parameters $(\alpha, \beta)=(2.26,0.65)$ due to the long simulation time and are also slightly lower compared with the analytical ones. Therefore, since a BER $\approx 10^{-9}$ has practical interest in FSO systems, our analytical expressions can serve as a reliable method to evaluate the BER performance of dual-hop links.

\section{Vi. CONClusions ANd Future Work}

In this paper, we examined the outage performance of a multihop FSO wireless system implemented by AF or DF relays. For these types of relays the outage probability was evaluated and analyzed. Furthermore, the average error probability is studied for the special case of a dual-hop system with a DF relay. Numerical examples were also provided for illustration purposes. The analysis presented in this paper can be easily extended to other turbulent channel models (e.g., $K$ or $I-K)$ and may contribute to a number of open issues for future investigation such as error rate analysis of FSO multihop systems with AF relays and how the delay in DF systems affects the overall performance of the system.

\section{REFERENCES}

[1] L. Andrews, R. L. Philips, and C. Y. Hopen, Laser Beam Scintillation with Applications. SPIE Press, 2001.

[2] M. A. Al-Habash, L. C. Andrews, and R. L. Philips, "Mathematical model for the irradiance probability density function of a laser beam propagating through turbulent media," Optical Engineering, vol. 40, pp. 1554-1562, Aug. 2001.

[3] X. Zhu and J. M. Kahn, "Free-space optical communication through atmospheric turbulence channels," IEEE Trans. Commun., vol. 50, pp. 1293-1300, Aug. 2002.

[4] - "Performance bounds for coded free-space optical communications through atmospheric turbulence channels," IEEE Trans. Commun., vol. 51, pp. 1233-1239, Aug. 2003.

[5] M. Uysal, S. M. Navidpour, and J. T. Li, "Error rate performance of coded free-space optical links over strong turbulence channels," IEEE Commun. Lett., vol. 8, pp. 635-637, Oct. 2004.

[6] M. Uysal and J. T. Li, "Error rate performance of coded free-space optical links over gamma-gamma turbulence channels," in Proc. of IEEE International Communications Conference (ICC'04), Paris, France, June 2004, pp. 3331-3335.

[7] A. S. Acampora and S. V. Krishnamurthy, "A broadband wireless access network based on mesh-connected free-space optical links," IEEE Personal Communications, vol. 6, pp. 62-65, Oct. 1999.

[8] J. Akella, M. Yuksel, and S. Kalyanaraman, "Error analysis of multihop free-space optical communication," in Proc. of IEEE Internatioanl Conference on Communications 2005 (ICC'05), Seoul, Korea, May 2005.

[9] M. O. Hasna and M. S. Alouini, "Outage probability of multihop transmission over Nakagami fading channels," IEEE Commun. Lett., vol. 7, pp. 216-218, May 2003.

[10] G. K. Karagiannidis, D. A. Zogas, N. C. Sagias, T. A. Tsiftsis, and P. T. Mathiopoulos, "Multihop communications with fixed-gain relays over generalized fading channels," in Proc. IEEE Global Telecommunications Conference (GLOBECOM '04), vol. 1, Dallas Texas, USA, Dec. 2004, pp. $36-40$.

[11] G. K. Karagiannidis, T. A. Tsiftsis, and R. K. Mallik, "Bounds for multihop relayed communications in Nakagami- $m$ fading," IEEE Trans. Commun., vol. 54, pp. 18-22, Jan. 2006.

[12] J. N. Laneman and G. W. Wornell, "Energy-efficient antenna sharing and relaying for wireless networks," in Proc. IEEE Wireless Communications and Networking Conf. (WCNC'00), Chicago, IL, Oct. 2000, pp. 7-12.

[13] I. S. Gradshteyn and I. M. Ryzhik, Table of Integrals, Series, and Products, 6th ed. New York: Academic, 2000.

[14] L. C. Andrews, R. L. Philips, and C. Y. Hopen, "Theory of optical scintillation," Journal of Optical Society of America A, vol. 16, no. 6 , pp. 1417-1429, June 1999 .

[15] V. S. Adamchik and O. I. Marichev, "The algorithm for calculating integrals of hypergeometric type functions and its realization in REDUCE system," in Proc. International Conference on Symbolic and Algebraic Computation, Tokyo, Japan, 1990, pp. 212-224.

[16] R. M. Gagliardi, Intoduction to Communications Engineering. New York, NY:: Wiley \& Sons, Inc., 1988.

[17] M. K. Simon and M.-S. Alouini, Digital Communication over Fading Channels, 2nd ed. New York: Wiley, 2005.

[18] J. G. Proakis, Digital Communications, 4th ed. New York: McGrawHill, 2001. 